Article

\title{
Campylobacter jejuni in Different Canine Populations: Characteristics and Zoonotic Potential
}

\author{
Maria-Leonor Lemos ${ }^{1}(\mathbb{D})$, Alexandra Nunes ${ }^{2,3,4}$, Massimo Ancora ${ }^{5}$, Cesare Cammà ${ }^{5}$ (D), \\ Paulo Martins da Costa ${ }^{1}$ (D) and Mónica Oleastro $2, *$ (D)
}

1 ICBAS-Institute of Biomedical Sciences Abel Salazar, University of Porto, 4050313 Porto, Portugal; up201305391@edu.icbas.up.pt (M.-L.L.); pmcosta@icbas.up.pt (P.M.d.C.)

2 Infectious Diseases Department, National Institute of Health Dr. Ricardo Jorge, 1649016 Lisbon, Portugal; alexandra.nunes@insa.min-saude.pt

3 Faculty of Veterinary Medicine, Lusófona University, 1300477 Lisboa, Portugal

4 CBIOS-Universidade Lusófona's Research Center for Biosciences \& Health Technologies, Campo Grande 376, 1749024 Lisbon, Portugal

5 Istituto Zooprofilattico Sperimentale dell'Abruzzo e del Molise “G. Caporale”, 64100 Teramo, Italy; m.ancora@izs.it (M.A.); c.camma@izs.it (C.C.)

* Correspondence: monica.oleastro@insa.min-saude.pt

Citation: Lemos, M.-L.; Nunes, A.; Ancora, M.; Cammà, C.; Costa, P.M.d.; Oleastro, M. Campylobacter jejuni in Different Canine Populations: Characteristics and Zoonotic Potential. Microorganisms 2021, 9 , 2231. https://doi.org/10.3390/ microorganisms 9112231

Academic Editor: Hirokazu Kimura

Received: 21 September 2021

Accepted: 25 October 2021

Published: 26 October 2021

Publisher's Note: MDPI stays neutral with regard to jurisdictional claims in published maps and institutional affiliations.

Copyright: (c) 2021 by the authors. Licensee MDPI, Basel, Switzerland. This article is an open access article distributed under the terms and conditions of the Creative Commons Attribution (CC BY) license (https:// creativecommons.org/licenses/by/ $4.0 /)$.

\begin{abstract}
With most epidemiological studies focused on poultry, dogs are often overlooked as a reservoir of Campylobacter, even though these animals maintain close daily contact with humans. The present study aimed to obtain a first insight into the presence and characteristics of Campylobacter spp. in different canine populations in Portugal, and to evaluate its zoonotic potential through genomic analysis. From a total of 125 rectal swabs collected from companion $(n=71)$ and hunting dogs $(n=54)$ living in two different settings, rural $(n=75)$ and urban $(n=50), 32$ Campylobacter spp. isolates were obtained. Four different Campylobacter species were identified by Multiplex PCR and MALDI-TOF mass spectrometry, of which Campylobacter jejuni $(n=14,44 \%)$ was overall the most frequently found species. Relevant resistance phenotypes were detected in C. jejuni, with $93 \%$ of the isolates being resistant to ciprofloxacin, $64 \%$ to tetracycline, and $57 \%$ to ampicillin, and three isolates being multi-drug-resistant. Comparison of the phenotypic and genotypic traits with human isolates from Portuguese patients revealed great similarity between both groups. Particularly relevant, the wgMLST analysis allowed the identification of isolates from human and dogs without any apparent epidemiological relationship, sharing high genetic proximity. Notwithstanding the limited sample size, considering the high genomic diversity of C. jejuni, the genetic overlap between human and dog strains observed in this study confirmed that the occurrence of this species in dogs is of public health concern, reinforcing the call for a One Health approach.
\end{abstract}

Keywords: Campylobacter spp.; dogs; whole genome sequencing; One Health

\section{Introduction}

Since 2005, campylobacteriosis is the most reported zoonotic disease in the European Union, mostly associated with Campylobacter jejuni and Campylobacter coli infections [1]. Being mainly a foodborne disease, it is frequently related to the consumption and handling of contaminated meat. Since $\sim 80 \%$ of human infections are associated with poultry, epidemiological studies and prevention programs have largely focused on broilers [2,3]. Despite that, other sources of infection, such as wild animals, environmental water, and pets have been described and should also be considered [4].

In dogs, Campylobacter upsaliensis and C. jejuni are the predominantly found species, and despite being mainly considered as commensal organisms, with a prevalence of around 50\% among subclinical animals, these are also described as pathogenic, especially in younger dogs, and are associated with clinical signs similar to those described in humans [5-7]. Owning a dog, particularly puppies, has been described as a risk factor 
for campylobacteriosis, and different studies around Europe have attributed 9-25\% of human cases to pets [6,8-12]. The fact that neither dogs nor humans are considered primary sources or amplifying hosts of Campylobacter and that the close sharing of a living environment exposes them to common sources of infection, limits the assessment of the exact transmission route [8], which could lead to a bias in the aforementioned values. Despite that, there is evidence for dog-human transmission [13,14].

The ease with which bacteria and their resistance genes can spread among human and animal populations forces an extensive approach-based on the One Health conceptwhen facing this kind of zoonosis [15]. Therefore, comprehensive studies are essential, using appropriate typing methods, which allow an efficient assessment of the relationship between different strains and hosts.

Since there was no previous study regarding Campylobacter spp. in dogs in Portugal, the present study aimed to obtain a first insight into the presence and characteristics of this microorganism in different canine populations, as well as to assess the zoonotic potential of the Campylobacter jejuni isolates through a genomic comparison with human isolates.

\section{Materials and Methods}

\subsection{Sample Selection and Collection}

To evaluate different canine populations, rectal swabs were collected from pet and hunting dogs, in two different settings, rural and urban.

Seventy-one samples from household pets were collected from September to December 2020 from a Veterinary Hospital of the University of Porto (urban area) $(n=50)$ and a Veterinary Clinic located in a rural area in the north of Portugal $(n=21)$. Rectal swab specimens were obtained from dogs presented for consultation $(n=39)$, in pre- and postsurgery care $(n=16)$ and hospitalized $(n=16)$, on a convenience basis. The study design, including its ethical aspects, was reviewed and approved in advance with the report number P330/2019/ORBEA by the Ethical Committee of ICBAS (ORBEA) and by the Portuguese Animal Welfare Authority (DGAV). The owners were asked to sign a consent form and fill out a short questionnaire about the animal and its living environment, signs of gastrointestinal illnesses, and any recent antibiotic treatment. Among the 71 sampled dogs (male $n=32$; female $n=39$ ), 12 presented with a recent history of diarrhea, and five of them were in the hospital for that reason.

Samples of hunting dogs were collected from boarhounds and small game hounds from the same rural area as the Veterinary Clinic. Regarding the boarhounds, on November 23,2020 , two weeks after the opening of the boar hunting season, 17 (male $n=9$; female $n=8$ ) rectal swabs were collected from the same pack. Concerning this group, 12 dogs had already been hunting that season and five had not still. A month after the first sampling campaign, after all the animals had been hunting in "montarias" (typical Iberian boardriven hunt), a second swab was collected from 15 of the initial 17 dogs sampled. Along with this boarhound pack, 22 (male $n=14$; female $n=8$ ) samples from small game hounds from the same area were obtained in November 2020 from three different packs $(n=8$; $n=11 ; n=3)$, totaling 54 specimens from hunting dogs. Regarding this group, none of the animals presented a history of gastrointestinal disease.

\subsection{Culture and Isolation of Campylobacter spp.}

Immediately after the rectal swab was performed, samples were placed into $10 \mathrm{~mL}$ of Bolton Broth (Bolton Broth with Bolton broth selective supplement, and 5\% Horse blood Lysed, Oxoid, Madrid, Spain). Inoculated broths were incubated for $48 \mathrm{~h}$, at $41.5^{\circ} \mathrm{C}$ under microaerophilic conditions generated by CampyGen ${ }^{\mathrm{TM}}$ (Oxoid). After the enrichment, $120 \mu \mathrm{L}$ of the suspension were placed in blood agar (BA) (Blood Agar: Trypto-casein soy agar, Biokar Diagnostics, supplemented with 5\% Horse Lysed blood, Oxoid) through a $0.65 \mu \mathrm{m}$ membrane filter (Nitrocellulose membrane filters, Whatman, Stuttgart, Germany) and subsequently incubated in the same conditions, for another 48-h period (ISO 10272:2006-1). 
Campylobacter-like colonies (about five per sample) were tested for oxidase (Oxidase Test Stick, Liofilchem, Roseto degli Abruzzi, Italy) and growth under aerobic conditions, and morphology and motility evaluated through phase-contrast microscopy. DNA was extracted from the suspected colonies by the boiling method.

\subsection{Isolate Identification}

A PCR Multiplex Campylobacter was performed as described by Yamazaki-Matsune and colleagues (2007) for Campylobacter genus confirmation and species identification, targeting C. jejuni, C. upsaliensis, C. coli, C. fetus, C. lari and C. hyointestinalis [16]. PCR products were analyzed by gel electrophoresis through a $1.5 \%$ (weight/volume) agarose (Agarose Ultrapure grade, NZYtech, Lisbon, Portugal) in $1 \times$ TBE buffer, stained with 1\% GreenSafe (GreenSafe Premium, NZYTech).

All the isolates confirmed as Campylobacter spp. by the Multiplex PCR, but whose species could not be identified by this method, were submitted to identification by matrix assisted laser desorption ionization-time of flight (MALDI-TOF) mass spectrometry (Vitek ${ }^{\circledR}$ MS, Oxoid).

\subsection{Antimicrobial Susceptibility Testing}

Antimicrobial susceptibility testing was performed, according to the Kirby-Bauer method. In Mueller-Hinton agar supplemented with 5\% defibrinated horse blood (Oxoid) and $\beta$-NAD (Sigma, Lisbon, Portugal), a $0.5 \mathrm{McFarland}$ suspension of bacterial culture in saline solution was inoculated and antimicrobial susceptibility testing was performed by disk diffusion, for the following drugs (charge of the disk): ciprofloxacin (5 $\mathrm{gg})$, erythromycin $(15 \mu \mathrm{g})$, tetracycline $(30 \mu \mathrm{g})$, gentamicin $(10 \mu \mathrm{g})$, ampicillin $(10 \mu \mathrm{g})$ and amoxicillin/clavulanic acid (30 $\mu \mathrm{g})$ (Oxoid).

The diameters of the inhibition zones were measured after a 24 -h incubation, at $41.5{ }^{\circ} \mathrm{C}$, in a microaerobic environment generated by Anoxomat (Advanced Instruments Inc., Königswinter, Germany). Results were interpretated following the European Committee on Antimicrobial Susceptibility Testing (EUCAST) [17] and the Comité de l'antibiogramme de la Société Française de Microbiologie cut-offs.

\subsection{Molecular Typing}

Molecular typing was performed using enterobacterial repetitive intergenic consensus PCR (ERIC-PCR), using as template the DNA extracted in the automated nucleic acid extraction platform eMAG ${ }^{\mathrm{TM}}$ (bioMérieux, Marcy-l'Étoile, France), and the primers described by Versalovic and colleagues (1991), ERIC-1R (5'-ATGTAAGCTCCTGGGGATTCAC-3') and ERIC-2 (5' - AAGTAAGTGACTGGGGTGAGCG-3') [18]. The PCR products were separated in $2 \%(w / v)$ agarose (Agarose Ultrapure grade, NZYtech) in $1 \times$ TBE buffer, stained with $1 \%$ GreenSafe (GreenSafe Premium, NZYTech).

\subsection{Whole Genome Sequencing and Bioinformatics Analysis}

Whole genome sequencing (WGS) was performed in 10 selected isolates of C. jejunirepresentative of the variety of ERIC profiles obtained-using the Illumina technology. Each sample was quantified with the Qubit fluorometer (Qubit ${ }^{\mathrm{TM}}$ DNA HS Assay, Life Technologies, Thermo Fisher Scientific Inc., Porto Salvo, Portugal). Library preparation was obtained using the Illumina DNA Prep kit (Illumina Inc., San Diego, CA, USA) according to the manufacturer's manual. The libraries prepared were loaded onto NextSeq 500/550 Mid Output Reagent Cartridge v2, 300 cycles kit (Illumina Inc.) and sequenced on an Illumina NextSeq 500 platform and generated $150 \mathrm{bp}$ paired-end reads.

Genomes were de novo assembled as previously described [19], using the INNUca v4.2.0 pipeline (https:/ / github.com/B-UMMI/INNUca, accessed on 2 February 2021) [20] that consists of several integrated modules for reads QA/QC, de novo assembly and post-assembly optimization steps. The obtained draft genome sequences (of $\sim 1.66 \mathrm{Mbp}$ in length split in no more than 41 contigs for ST-22 strains and of $\sim 1.76 \mathrm{Mbp}$ in length split 
in no more than 62 contigs for ST-6461 strains) were annotated with the RAST server v2.0 (http: / / rast.nmpdr.org/, accessed on 8 February 2021) [21]. Raw sequence reads used in the present study were deposited in the European Nucleotide Archive (ENA) under the study accession number PRJEB44937.

In silico Multilocus Sequence Typing (MLST), extended Multilocus Sequence Typing (eMLST) and flaA short variable region (flaA svr) typing was determined at the PubMLST platform (https:/ / pubmlst.org/, accessed on 12 February 2021).

In order to understand the phylogenetic positioning of the Portuguese (PT) isolates from dogs, other ST-6461 $(n=91)$ and ST-22 $(n=83)$ (sharing the same flaA svr type of the PT dogs), strains from different geographic regions and sources (Tables S1 and S2), with available genomes, archived in BIGSdb (accessed on 15 February 2021), were selected, and their paired reads were downloaded from ENA and de novo assembled as described above. In addition, from 48 Portuguese clinical strains collected in 2020, with available genomes, one ST-6461 strain and four ST-22 strains were selected and included in the phylogenetic analysis. The genetic relatedness among strains was evaluated through comparative gene-by-gene analysis using the publicly available C. jejuni INNUENDO cgMLST and wgMLST Schemas composed by 678 and 2795 loci, respectively [22]. For all genomes, allele calling was performed against both schemas, using chewBBACA v2.0.16 [23] with default parameters. Exact and inferred matches were used to construct an allelic profile matrix, where other allelic classifications (see https:/ / github.com/B-UMMI/chewBBACA/wiki, accessed on 1 September 2021) were assumed as "missing" loci. Only profiles with no more than $2 \%$ of missing loci in either cgMLST were used for subsequent phylogenetic inferences [22]. Minimum spanning trees (MST) were constructed by taking advantage of the goeBURST algorithm [24] implemented in the PHYLOViZ online 2.0 Beta version (http: / / online2.phyloviz.net, accessed on 25 February 2021) web-based tool [25], based on $100 \%$ shared loci between all isolates (i.e., shared-genome MLST). This allows to increase the resolution power for cluster analysis by maximizing the shared genome in a dynamic manner, i.e., for each sub-set of strains under comparison, the maximum number of shared loci between them is automatically used for tree construction. Whenever it was useful, allelic distances were expressed as percentages of allele differences (AD), expressed as the number of allelic differences over the total number of shared loci under comparison.

\subsection{Data Analysis}

Data regarding the variables: setting, age, sex, and display of diarrhea underwent univariate analysis for each canine population, using Fisher's exact test given the small sample size. Campylobacter spp. status (positive or negative) was used as the dependent variable. A $p$-value of $<0.05$ was considered significant.

\section{Results}

\subsection{Frequency and Distribution of Campylobacter spp.}

Among the 125 rectal swabs collected from 110 dogs, 26 (20.8\%; 95\% confidence interval $(\mathrm{CI})=14.6-28.7 \%$ ) had a positive culture for Campylobacter spp., and a total of 32 isolates was obtained (Table 1). There was a higher frequency in hunting dogs, with $19 / 54(35.2 \% ; 95 \% \mathrm{CI}=23.8-48.5 \%)$ of positive samples, than in companion dogs, with Campylobacter spp. being isolated in $7 / 71(9.9 \% ; 95 \% \mathrm{CI}=4.9-19.0 \%)$ of the samples collected; this difference was statistically significant $(p<0.05)$. Regarding the distribution of Campylobacter species, $C$. jejuni $(n=14)$ was the predominant species, with most isolates $(12 / 14)$ being obtained from hunting dogs, and the remaining (2/14) from dogs presented for consultation, one at the Veterinary Clinic and the other at the Veterinary Hospital; only the latter showed clinical signs. The second most isolated species was Campylobacter lari with 13 isolates obtained from healthy boarhounds. Four C. upsaliensis isolates were found, three of them from diarrheic animals presented at the Veterinary Hospital, and one from a non-diarrheic dog sampled at the Veterinary Clinic. Finally, a single C. coli isolate was detected in a non-diarrheic dog at the Veterinary Clinic. Mixed colonization was observed 
in six animals, five of them simultaneously presenting C. jejuni and C. lari and another one carrying two different strains of $C$. jejuni. The only significant association observed was between the presence of $C$. jejuni and $C$. upsaliensis and the display of diarrhea in companion dogs from the urban setting $(p<0.05)$. Apart from the apparent association with clinical signs, no other surveyed variable showed significant differences $(p<0.05)$.

Table 1. Characteristics of the samples collected $(n=125)$ and species distribution among Campylobacter isolates $(n=32)$, according to canine populations.

\begin{tabular}{|c|c|c|c|c|c|c|c|c|}
\hline \multirow{2}{*}{$\begin{array}{c}\text { N-Total } \\
\text { [n1-Companion/n2-Hunting] }\end{array}$} & \multicolumn{2}{|c|}{ Gender } & \multicolumn{2}{|c|}{ Age } & \multicolumn{2}{|c|}{ Diarrhea } & \multicolumn{2}{|c|}{ Setting } \\
\hline & Male & Female & $0-2$ & $>2$ & Yes & No & Urban & Rural \\
\hline N. dogs & $\begin{array}{c}55 \\
{[32 / 23]}\end{array}$ & $\begin{array}{c}55 \\
{[39 / 16]}\end{array}$ & $\begin{array}{c}42 \\
{[23 / 19]}\end{array}$ & $\begin{array}{c}68 \\
{[44 / 24]}\end{array}$ & $\begin{array}{c}12 \\
{[12 / 0]}\end{array}$ & $\begin{array}{c}98 \\
{[59 / 39]}\end{array}$ & $\begin{array}{c}50 \\
{[50 / 0]}\end{array}$ & $\begin{array}{c}60 \\
{[21 / 39]}\end{array}$ \\
\hline N. samples & $\begin{array}{c}63 \\
{[32 / 31]}\end{array}$ & $\begin{array}{c}62 \\
{[39 / 23]}\end{array}$ & $\begin{array}{c}47 \\
{[23 / 24]}\end{array}$ & $\begin{array}{c}78 \\
{[44 / 34]}\end{array}$ & $\begin{array}{c}12 \\
{[12 / 0]}\end{array}$ & $\begin{array}{c}113 \\
{[59 / 54]}\end{array}$ & $\begin{array}{c}50 \\
{[50 / 0]}\end{array}$ & $\begin{array}{c}75 \\
{[21 / 54]}\end{array}$ \\
\hline \multicolumn{9}{|l|}{ Distribution by species } \\
\hline C. jejuni & $\begin{array}{c}10 \\
{[2 / 8]}\end{array}$ & $\begin{array}{c}4 \\
{[0 / 4]}\end{array}$ & $\begin{array}{c}4 \\
{[2 / 2]}\end{array}$ & $\begin{array}{c}10 \\
{[0 / 10]}\end{array}$ & $\begin{array}{c}1 \\
{[1 / 0]}\end{array}$ & $\begin{array}{c}13 \\
{[1 / 12]}\end{array}$ & $\begin{array}{c}1 \\
{[1 / 0]}\end{array}$ & $\begin{array}{c}13 \\
{[1 / 12]}\end{array}$ \\
\hline C. upsaliensis & $\begin{array}{c}2 \\
{[2 / 0]}\end{array}$ & $\begin{array}{c}2 \\
{[2 / 0]}\end{array}$ & $\begin{array}{c}2 \\
{[2 / 0]}\end{array}$ & $\begin{array}{c}2 \\
{[2 / 0]}\end{array}$ & $\begin{array}{c}3 \\
{[3 / 0]}\end{array}$ & $\begin{array}{c}1 \\
{[1 / 0]}\end{array}$ & $\begin{array}{c}3 \\
{[3 / 0]}\end{array}$ & $\begin{array}{c}1 \\
{[1 / 0]}\end{array}$ \\
\hline C. lari & $\begin{array}{c}6 \\
{[0 / 6]}\end{array}$ & $\begin{array}{c}7 \\
{[0 / 7]}\end{array}$ & $\begin{array}{c}5 \\
{[0 / 5]}\end{array}$ & $\begin{array}{c}8 \\
{[0 / 8]}\end{array}$ & $\begin{array}{c}0 \\
{[0 / 0]}\end{array}$ & $\begin{array}{c}13 \\
{[0 / 13]}\end{array}$ & $\begin{array}{c}0 \\
{[0 / 0]}\end{array}$ & $\begin{array}{c}13 \\
{[0 / 13]}\end{array}$ \\
\hline C. coli & $\begin{array}{c}1 \\
{[1 / 0]}\end{array}$ & $\begin{array}{c}0 \\
{[0 / 0]}\end{array}$ & $\begin{array}{c}1 \\
{[1 / 0]}\end{array}$ & $\begin{array}{c}0 \\
{[0 / 0]}\end{array}$ & $\begin{array}{c}0 \\
{[0 / 0]}\end{array}$ & $\begin{array}{c}1 \\
{[1 / 0]}\end{array}$ & $\begin{array}{c}0 \\
{[0 / 0]}\end{array}$ & $\begin{array}{c}1 \\
{[1 / 0]}\end{array}$ \\
\hline Total & $\begin{array}{c}19 \\
{[5 / 14]}\end{array}$ & $\begin{array}{c}13 \\
{[2 / 11]}\end{array}$ & $\begin{array}{c}12 \\
{[5 / 7]}\end{array}$ & $\begin{array}{c}20 \\
{[2 / 18]}\end{array}$ & $\begin{array}{c}4 \\
{[4 / 0]}\end{array}$ & $\begin{array}{c}28 \\
{[3 / 25]}\end{array}$ & $\begin{array}{c}4 \\
{[4 / 0]}\end{array}$ & $\begin{array}{c}28 \\
{[3 / 25]}\end{array}$ \\
\hline
\end{tabular}

Substantial differences were observed when comparing the two sampling moments of the boarhounds (Table S3). Regarding the first sampling event, C. lari was the most frequently identified Campylobacter species (7/9) followed by C. jejuni (2/9), both obtained from animals that had recently been hunting. In the second sampling moment, performed a month later, after all the dogs had been hunting, there was an increase in the number of isolates obtained $(n=14)$, especially regarding $C$. jejuni, which became the most frequently identified species (8/14). Tendentially, dogs held the same Campylobacter spp. status (positive or negative) in both moments. Only in two animals with a positive status in the first sampling time, no isolate was obtained in the second moment, and regarding the dogs with a negative status in the first sample, only one later tested positive. It should be noted that, in the second collection, most of the animals presenting Campylobacter had mixed colonization $(6 / 8)$, a fact not observed in the first moment.

\subsection{Campylobacter jejuni}

Considering that $C$. jejuni was the species more frequently found in this study and being the main responsible for human infection, we focused our study on this species. Of the total C. jejuni isolates $(n=14)$, only seven were identified at species level by PCR Multiplex and the identification of the remaining was carried out by MALDI-TOF. All $C$. jejuni isolates not identified by the multiplex PCR belonged to ST-22. Several mismatches were detected in the primer annealing sequence of the target gene (cj04141) preventing the species identification of these isolates (data not shown). Antimicrobial susceptibility testing revealed a high proportion of $C$. jejuni isolates resistant to ciprofloxacin (93\%), tetracycline $(64 \%)$, and ampicillin $(57 \%)$. No resistance to erythromycin, gentamicin, or amoxicillin/clavulanic acid was observed. Altogether, five different drug resistance profiles of $C$. jejuni were found, with three isolates being multidrug resistant (ciprofloxacin, tetracycline, and ampicillin). 
ERIC-PCR fingerprints of the 13 isolates tested showed seven different profiles (Figure 1), which could be grouped into four clusters based on their similarities; the first cluster (C1) included a single isolate from the boarhounds; the second one (C2) comprised three isolates from hunting dogs: one from the pack of boarhounds and two from the same pack of small game hounds. Cluster 3 (C3) consisted of three isolates obtained in the second sample collection of boarhounds, and finally, cluster 4 (C4) comprised five isolates from the boarhounds and one from the Veterinary Hospital.

To further explore genetic diversity, 10 isolates, representative of the variety of ERIC profiles obtained, were subjected to WGS. The in silico MLST typing showed that the 10 isolates were distributed in five STs: ST-22 $(n=4)$, ST-6461 $(n=3)$, ST-48 $(n=1)$, ST-148 $(n=1)$, and ST-8569 $(n=1)$. The in silico flaA short variable region (svr) identified eight different alleles, with three isolates displaying two flaA svr alleles. A correlation between flaA svr and MLST typing was observed regarding ST-22, as all isolates had the same allele (flaA-161). The same was not observed among those belonging to the ST-6461 where a flaA svr variety was detected, with the isolates belonging to flaA-67, flA-49/395, and $f l A-49$.

In general, the clustering of the isolates was highly consistent between different typing methods (Table 2); in fact, except for C2, which hosted two different STs (ST-8569 and ST-148), each ERIC-PCR cluster was correlated with a specific ST (C1: ST-48, C3: ST-6461 and C4: ST-22).

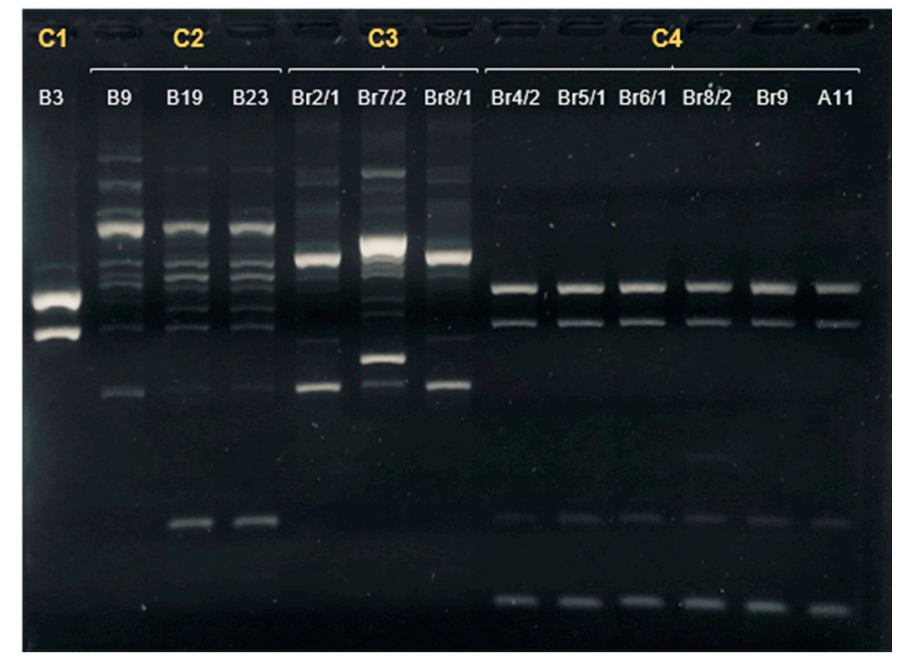

Figure 1. ERIC-PCR fingerprints of Campylobacter jejuni isolated in dogs. The ID of the sample is presented in white. The letter and numbers in yellow represent the cluster assigned to the profile.

Table 2. ERIC-PCR, Sequence type, Clonal complex and flaA svr of Campylobacter jejuni isolates from dogs studied by whole genome sequence.

\begin{tabular}{|c|c|c|c|c|c|c|}
\hline Sample & Date of Isolation & Group & ERIC-PCR Cluster & MLST Sequence Type & Clonal Complex & flaA svr \\
\hline A11 & $24 / 09 / 2020$ & Companion/Urban & 4 & 22 & 22 & 161 \\
\hline B3 & $23 / 10 / 2020$ & Hunting/Rural & 1 & 48 & 48 & $32 ; 102$ \\
\hline B9 & $23 / 10 / 2020$ & Hunting/Rural & 2 & 8569 & 179 & 571 \\
\hline B19 & $6 / 11 / 2020$ & Hunting/Rural & 2 & 148 & 21 & $36 ; 36$ \\
\hline $\mathrm{Br} 2 / 1$ & $20 / 11 / 2020$ & Hunting/Rural & 3 & 6461 & 353 & 67 \\
\hline $\mathrm{Br} 4 / 2$ & $20 / 11 / 2020$ & Hunting/Rural & 4 & 22 & 22 & 161 \\
\hline $\mathrm{Br} 7 / 2$ & $20 / 11 / 2020$ & Hunting/Rural & 3 & 6461 & 353 & $49 ; 395$ \\
\hline Br8/1 & $20 / 11 / 2020$ & Hunting/Rural & 3 & 6461 & 353 & 49 \\
\hline $\mathrm{Br} 8 / 2$ & $20 / 11 / 2020$ & Hunting/Rural & 4 & 22 & 22 & 161 \\
\hline $\mathrm{Br} 9$ & $20 / 11 / 2020$ & Hunting/Rural & 4 & 22 & 22 & 161 \\
\hline
\end{tabular}

When analyzing the C. jejuni isolates from boarhounds, a diversity of C. jejuni strains was observed among the different animals from the same pack, and among the same animals in the two sampling events (Table S3). The two isolates obtained in the first 
moment belonged to two different STs (ST-48 and ST-8569) that were not identified in the second sampling moment. In this case, isolates were distributed by ST-22 $(n=5)$ and ST-646 $(n=3)$, with one dog harboring C. jejuni isolates from two different STs.

\subsection{Comparison with Human Isolates}

The genomes of the dog strains from the two most frequent STs (ST-6461, $n=3$; ST-22, $n=4)$ were analyzed together with those of strains from humans isolated in Portugal, during 2020 from the same STs (ST-6461, $n=1$; ST-22, $n=4$; Tables S1 and S2, respectively), through ERIC-PCR, eMLST and wgMLST. Similar genomic relationships were pointed by all methods used, with a high degree of congruency between the results of ERIC-PCR (Figure 2) and eMLST (Table S4). The analysis of eMLST allowed the detection of differences between isolates from the same ST. Regardless of the source, these differences were also observed in the ERIC profile, as variations in a small number of loci could be associated with minor changes in the ERIC profile (Figure 2).

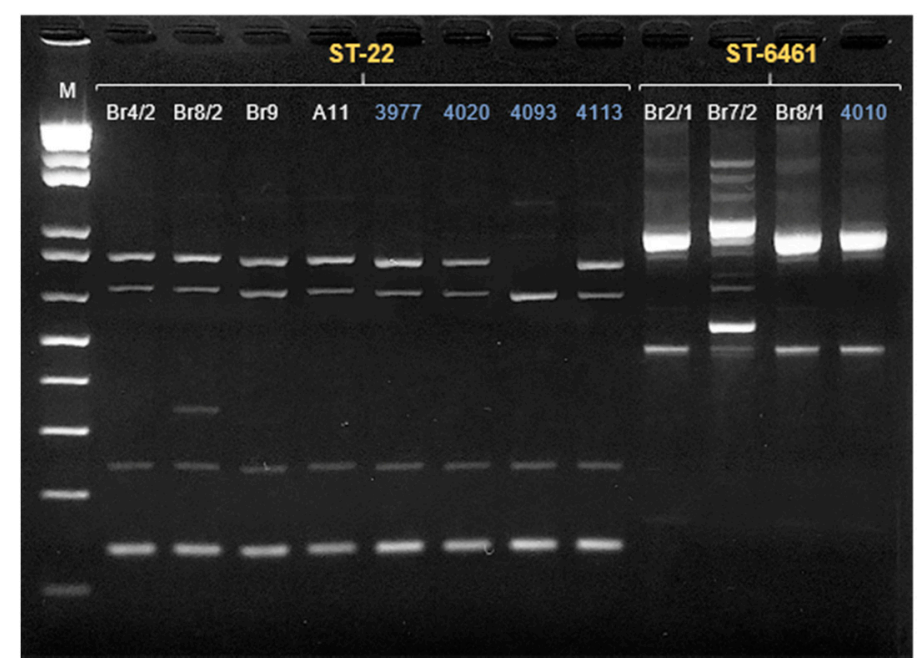

Figure 2. ERIC-PCR fingerprints of Campylobacter jejuni ST-22 and ST-6461 (presented in yellow) in a $2 \%$ agarose gel. Lane M, $1 \mathrm{Kbp}$ Plus ladder (Invitrogen, Waltham, MA, USA). The ID of the sample is presented in white in the case of the dog isolates and in blue in the human isolates. Finally, a comparative gene-by-gene analysis was performed between PT isolates and other ST-6461 $(n=91)$ and ST-22 $(n=83)$ with available genomes from different regions and sources (Supplementary Tables S1 and S2), with the results represented by minimum spanning trees (MSTs) (Figures 3 and 4).

All three dog ST-6461 PT isolates exhibited the same wgMLST profile, and were genetically closer to the PT human isolate, composing a genetic cluster with only 14 allelic differences between them (Figure 3A). When compared with the remainder ST-6461 strains, a median of 38 allelic differences is seen (Figure 3B).

Regarding ST-22 (Figure 4), PT isolates were also found to be genetically closer to each other ( $<13 \mathrm{AD})$, composing an independent cluster that distances by at least 24 alleles from the remaining ST-22 isolates. The only exception was one PT human isolate (4093) that, differing by 281 alleles, integrated a completely distinct cluster. Interestingly, two dog isolates (Br4/2 and Br9) shared the same allelic profile as a human isolate (4020). 
A

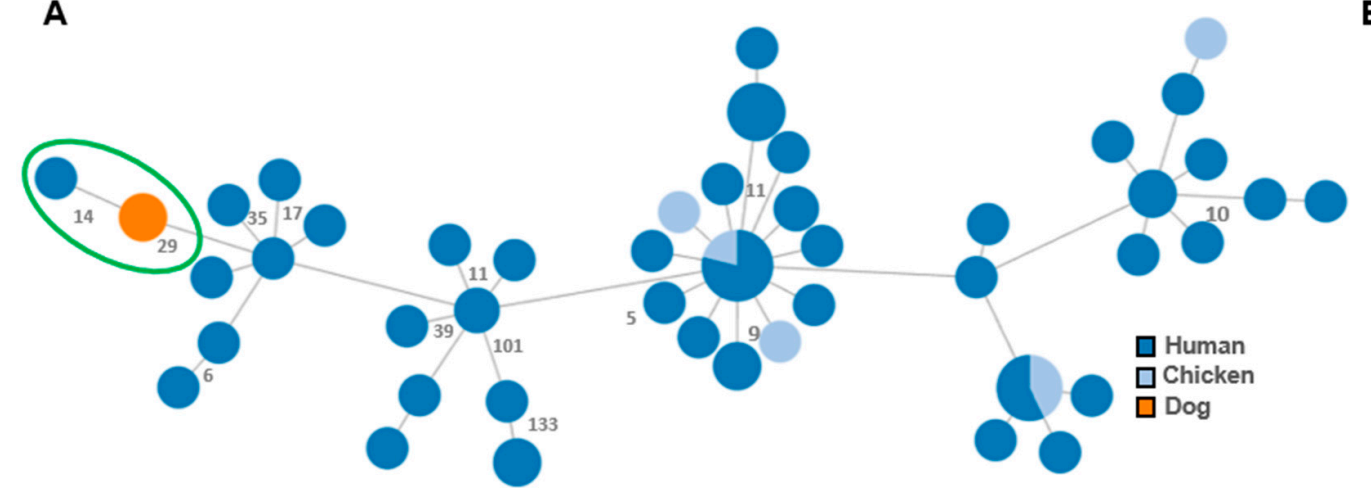

B

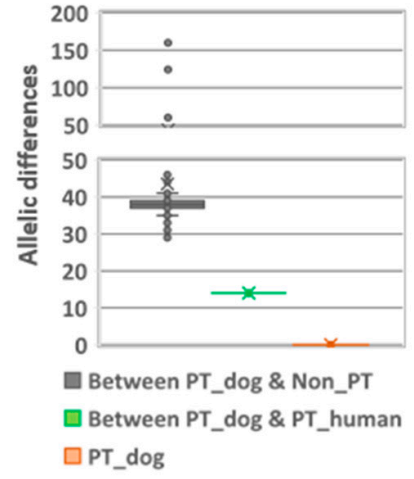

Figure 3. Genomic relatedness among Campylobacter jejuni ST-6461 strains based on a dynamic gene-by-gene approach using a wgMLST schema with 2795 loci. (A) The Minimum spanning tree was constructed using the goeBURST algorithm implemented in the PHYLOViZ online platform and is based on the allelic diversity found among the 1011 genes shared by $100 \%$ of the validated strains. Filled circles (nodes) represent unique allelic profiles, and are colored according to strains' source (human, chicken, or dog). The size of nodes is proportional to the number of isolates it represents. Portuguese strains are marked with a green circle: three isolates from dogs (PT_Cj-Br2/1, PT_Cj-Br7/2, PT_Cj-Br8/1) and one from human (PT_Cj-4010). The numbers in grey on the connecting lines represent the allele differences (AD) between strains. For better visualization, only $\mathrm{AD} \geq 5$ are shown. (B) Box plot depicting the $\mathrm{AD}$ (including the median values) within and between PT strains from dogs and the remainder.

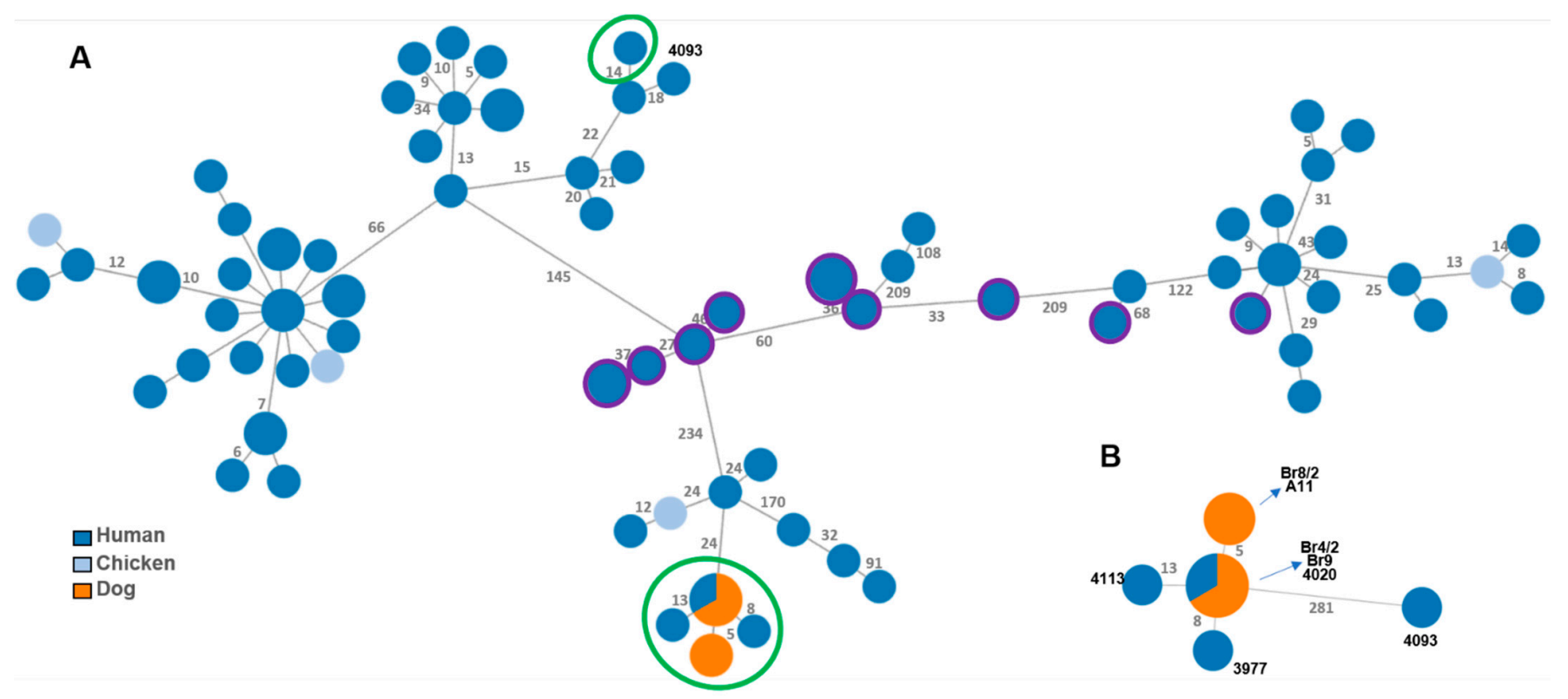

Figure 4. Phylogeny of Campylobacter jejuni ST-22 strains based on a dynamic gene-by-gene approach using a wgMLST schema with 2477 loci. (A) The initial MST was constructed based on the allelic diversity found among the 924 genes shared by $100 \%$ of the validated strains. Portuguese strains are marked with green circles: four from dogs (A11, Br8/2, Br4/2, Br9) and four from humans $(3977,4020,4093,4113)$; US strains are marked with purple outer rings; the remaining strains are from the UK. (B) Sub-MST reconstruction based on the allelic diversity found among the 953 genes shared by all validated PT strains (ID strains in bold). For both panels, trees were constructed using the goeBURST algorithm implemented in the PHYLOViZ Online platform. Filled circles (nodes) representing unique allelic profiles are colored according to strains' source (human, chicken or dog). The size of nodes is proportional to the number of isolates it represents. The numbers in grey on the connecting lines represent the allele differences (AD) between strains. For better visualization, only AD $\geq 5$ are shown. 


\section{Discussion}

All Campylobacter species found in this study were previously reported in dogs $[6,26]$. Although a significantly higher frequency was observed in hunting dogs, these results should be viewed with caution and assumptions should not be made due to the small sample size and the second sampling in boarhounds. Despite the small sample size, in companion dogs from the urban setting, an association between diarrhea and pathogenic species of Campylobacter was found as described in the literature [27,28].

Regarding the detection of mixed colonization in boarhounds, the fact that the second samples were obtained further along in the hunting season, when the animals had already been exposed to a greater diversity of environments and animals, could possibly justify this observation.

Besides being the only species detected in all groups, C. jejuni was the most frequently isolated species in this study. These results are relevant from a public health point of view, since $C$. jejuni is the leading cause of human infections [4].

The fact that none of the $C$. jejuni isolates belonging to the ST-22 (6/14) could be identified by the Multiplex PCR is also relevant. This highlights the value of a careful choice of molecular targets that allows a generic detection of isolates and correct species identification. The unsuccessful PCR detection can greatly impact the diagnosis of infection, especially in cases where a direct molecular diagnosis is performed in the biological sample. This may lead to an oversight of certain Campylobacter strains, as could be the case with this ST, highly prevalent in our dog isolates.

The predominant Campylobacter species isolated in dogs are C. jejuni and C. upsaliensis, however, there are some divergences between studies on which is the most prevalent $[26,27,29]$. The fact that the classic diagnostic methods have been optimized aiming at $C$. jejuni and C. coli means that they are often not suitable for the isolation of more fastidious species, which are frequently susceptible to the antibiotics present in the selective media and may need different temperatures or times of incubation [30,31]. In this study, in addition to using an incubation temperature of $41.5^{\circ} \mathrm{C}$, higher than the optimal temperature for $C$. upsaliensis $\left(37^{\circ} \mathrm{C}\right)$, the high concentration of Cefoperazone $(20 \mathrm{mg} / \mathrm{L})$ in the enrichment broth (above the previously described MIC for the majority of this species [32]), could explain why the number of isolates obtained was lower than the usually reported $[6,29,33,34]$.

In dogs, C. coli is reportedly less frequent compared to C. jejuni $[6,35]$ and C. upsaliensis [6], this fact is in line with the results from this study, where only one isolate was obtained. Regarding C. lari, despite the high number of isolates found in this study, they were all obtained from the same group of dogs (boarhounds), the majority from the same animals sampled twice. This can thus form a bias that may explain the unusually high prevalence, as in dogs the prevalence reported is usually very low (1-2\%) [6].

Regarding antimicrobial resistance, previous studies in dogs have reported resistance to ciprofloxacin (9-58\%), tetracycline (12-32\%), and erythromycin to a lesser extent $(0-12 \%)[7,36-38]$. Although no resistance to erythromycin was observed in this study, higher resistance rates to ciprofloxacin and tetracycline were found $(93 \%$ and $64 \%$, respectively), and the results are very similar to those recently reported by EFSA regarding C. jejuni from humans in Portugal, where rates of $93 \%$ resistance to ciprofloxacin and $78 \%$ to tetracycline were described [4]. This highlights the dissemination of antimicrobial resistance between different hosts, reinforcing the need for a comprehensive approach to this problem.

When analyzing the isolates from boarhounds, as it was possible to confirm with phenotypic (antimicrobial susceptibility) and genotypic (ERIC-PCR and WGS) assessment, there was a high diversity of $C$. jejuni strains between animals from the same pack and between the same animals in the two sampling events (one month apart). Along with the fact that the bacterium was only isolated from animals that had recently been hunting, this could possibly mean that there is a higher risk of transmission through the environment (while hunting) than between dogs or through feeding, inside the kennel. For this to be 
confirmed, further studies should be done, not only including more sampling events over time but also covering other boarhound packs.

Among the five different STs identified in the dog isolates, four of them (ST-22; ST-48; ST-148 and ST-6461) represented STs widely found in humans, suggesting a genetic proximity between the isolates present in both species. This relationship becomes even more relevant through wgMLST, where the PT isolates from ST-22 and ST-6461 formed high proximity clusters with minimal allelic differences.

The genetic proximity observed between samples of the two different hosts could not be associated with any apparent epidemiologic relationship, as the same wgMLST allelic profile was obtained in a human sample collected in July 2020, $380 \mathrm{~km}$ away from the boarhound sample collected in November 2020. The same was observed regarding different canine populations, as the same wgMLST allelic profile was obtained from a sample of a companion dog in an urban area, collected in September 2020, and from a sample of a boarhound living more than $100 \mathrm{~km}$, in November 2020.

Four different scenarios can justify the presence of identical strains in humans and dogs, as previously reviewed by Mughini-Gras and colleagues (2013). They can both become infected from the same source or from different sources carrying the same strain, humans can become infected from dogs, or dogs can become infected by humans. Unlike the study of Mughini-Gras, where there was a known close proximity between both hosts (comparison between dogs and their owners), in the present study, there was no apparent relationship between the human and dog samples. As so, both hosts becoming infected by different sources carrying the same strain would best seem to explain these findings, even considering the genetic diversity of Campylobacter and the geographic distribution of the samples. Thus, this study raises questions regarding the distribution of Campylobacter strains and the transmissibility between the two hosts.

Besides being the most common among dogs, in this study, ST-22 presented the highest genetic similarity with the human isolates. In addition, this particular ST has been highly associated with chronic complications such as the Guillain-Barre syndrome, being overrepresented amongst patients with this post-infection complication [39-41]. Considering that this ST is rarely found in poultry, the most common animal source of C. jejuni, $[39,42,43]$, dogs could be a more relevant reservoir than currently considered.

Improved detection and control of clinically relevant Campylobacter species will be achieved through an integrated One Health approach that includes an appropriate selection of typing techniques. ERIC-PCR, which in this study revealed a strong correlation with MLST, seems to be a very suitable option for routine genotyping analysis, although it still needs optimization and validation with a greater number of isolates. Given its effortlessness and low cost, this technique may be of special interest regarding sources of Campylobacter not normally studied, as it is the case with dogs.

In conclusion, despite being the first insight into Campylobacter spp. in dogs in Portugal, this study revealed surprising genetic proximity (evident through wgMLST) between the C. jejuni isolates obtained in dogs and those isolated from geographically distant humans, without any apparent epidemiological relationship. Thus, regardless of the exact transmission route, which is of greater interest to determine, the overlap of Campylobacter strains between two hosts with ever closer contact in today's society, requires an in-depth study to assess the real impact that this relationship may represent in campylobacteriosis.

Supplementary Materials: The following are available online at https:/ / www.mdpi.com/article/10 .3390 /microorganisms9112231/s1, Table S1: Genomes from Campylobacter jejuni ST-6461 included in the phylogenetic analysis, Table S2: Genomes from Campylobacter jejuni ST-22 included in the phylogenetic analysis., Table S3: Summary of the results from both sampling events from the boarhounds ( $n=17$ dogs), Table S4: Extended MLST and flaA svr of Campylobacter jejuni ST-22 and ST-6461 isolates.

Author Contributions: Conceptualization, M.-L.L., P.M.d.C. and M.O.; methodology, M.-L.L., A.N, M.A. and C.C.; software, A.N.; writing—original draft preparation, M.-L.L., P.M.d.C. and M.O.; 
writing-review and editing M.-L.L., P.M.d.C. and M.O., A.N. and C.C.; project administration, P.M.d.C. and M.O.; funding acquisition, M.O. All authors have read and agreed to the published version of the manuscript.

Funding: This research was partially funded by the Italian Ministry of Health, grant No IZS AM 02/19 RC.

Institutional Review Board Statement: Ethical review and approval were waived for this study, due to collection of animal samples.

Informed Consent Statement: Not applicable.

Conflicts of Interest: The authors declare no conflict of interest.

\section{References}

1. EFSA and ECDC (European Food Safety Authority and European Centre for Disease Prevention and Control). The European Union One Health 2019 Zoonoses Report. EFSA J. 2021, 19, e06406. [CrossRef]

2. Mullner, P.; Spencer, S.E.F.; Wilson, D.J.; Jones, G.; Noble, A.D.; Midwinter, A.C.; Collins-Emerson, J.M.; Carter, P.; Hathaway, S.; French, N.P. Assigning the source of human campylobacteriosis in New Zealand: A comparative genetic and epidemiological approach. Infect. Genet. Evol. 2009, 9, 1311-1319. [CrossRef]

3. EFSA (European Food Safety Authority). Scientific Opinion on Campylobacter in broiler meat production: Control options and performance objectives and/or targets at different stages of the food chain. EFSA J. 2011, 9, 2105. [CrossRef]

4. EFSA and ECDC (European Food Safety Authority and European Centre for Disease Prevention and Control). The European Union Summary Report on Antimicrobial Resistance in zoonotic and indicator bacteria from humans, animals and food in 2018/2019. EFSA J. 2021, 19. [CrossRef]

5. Acke, E. Campylobacteriosis in dogs and cats: A review. N. Z. Vet. J. 2018, 66, 221-228. [CrossRef]

6. Iannino, F.; Salucci, S.; Di Donato, G.; Badagliacca, P.; Vincifori, G.; Di Giannatale, E. Campylobacter and antimicrobial resistance in dogs and humans: "One health" in practice. Vet. Ital. 2019, 55, 203-220. [CrossRef]

7. Acke, E.; McGill, K.; Golden, O.; Jones, B.R.; Fanning, S.; Whyte, P. Prevalence of thermophilic Campylobacter species in household cats and dogs in Ireland. Vet. Rec. 2009, 164, 44-47. [CrossRef] [PubMed]

8. Mughini-Gras, L.; Smid, J.H.; Wagenaar, J.A.; Koene, M.G.J.; Havelaar, A.H.; Friesema, I.H.M.; French, N.P.; Flemming, C.; Galson, J.D.; Graziani, C.; et al. Increased risk for Campylobacter jejuni and C. coli infection of pet origin in dog owners and evidence for genetic association between strains causing infection in humans and their pets. Epidemiol. Infect. 2013, 141, 2526-2535. [CrossRef]

9. Rosner, B.M.; Schielke, A.; Didelot, X.; Kops, F.; Breidenbach, J.; Willrich, N.; Gölz, G.; Alter, T.; Stingl, K.; Josenhans, C.; et al. A combined case-control and molecular source attribution study of human Campylobacter infections in Germany, 2011-2014. Sci. Rep. 2017, 7, 5139. [CrossRef]

10. Thépault, A.; Rose, V.; Quesne, S.; Poezevara, T.; Béven, V.; Hirchaud, E.; Touzain, F.; Lucas, P.; Méric, G.; Mageiros, L.; et al. Ruminant and chicken: Important sources of campylobacteriosis in France despite a variation of source attribution in 2009 and 2015. Sci. Rep. 2018, 8, 9305. [CrossRef]

11. Kittl, S.; Heckel, G.; Korczak, B.M.; Kuhnert, P. Source attribution of human Campylobacter isolates by MLST and fla-typing and association of genotypes with quinolone resistance. PLOS ONE 2013, 8, e81796. [CrossRef]

12. Mughini-Gras, L.; Pijnacker, R.; Coipan, C.; Mulder, A.C.; Fernandes Veludo, A.; de Rijk, S.; van Hoek, A.H.A.M.; Buij, R.; Muskens, G.; Koene, M.; et al. Sources and transmission routes of campylobacteriosis: A combined analysis of genome and exposure data. J. Infect. 2021, 82, 216-226. [CrossRef]

13. Wolfs, T.F.W.; Duim, B.; Geelen, S.P.M.; Rigter, A.; Thomson-carter, F.; Wagenaar, J.A. Neonatal Sepsis by Campylobacter jejuni: Genetically Proven Transmission from a Household Puppy. Clin. Infect. Dis. 2001, 32, 97-99. [CrossRef]

14. Damborg, P.; Olsen, K.E.P.; Nielsen, E.M.; Guardabassi, L. Occurrence of Campylobacter jejuni in Pets Living with Human Patients Infected with C. jejuni. J. Clin. Microbiol. 2004, 42, 1363-1364. [CrossRef]

15. McEwen, S.A.; Collignon, P.J. Antimicrobial Resistance: A One Health Perspective. Antimicrob. Resist. Bact. Livest. Companion Anim. 2018, 6, 521-547. [CrossRef]

16. Yamazaki-Matsune, W.; Taguchi, M.; Seto, K.; Kawahara, R.; Kawatsu, K.; Kumeda, Y.; Kitazato, M.; Nukina, M.; Misawa, N.; Tsukamoto, T. Development of a multiplex PCR assay for identification of Campylobacter coli, Campylobacter fetus, Campylobacter hyointestinalis subsp. hyointestinalis, Campylobacter jejuni, Campylobacter lari and Campylobacter upsaliensis. J. Med. Microbiol. 2007, 56, 1467-1473. [CrossRef]

17. The European Committee on Antimicrobial Susceptibility Testing. Breakpoint Tables for Interpretation of MICs and Zone Diameters. Version 11.0. 2021. Available online: http:/ / www.eucast.org (accessed on 1 September 2021).

18. Versalovic, J.; Koeuth, T.; Lupski, J.R.; Plaza, O.B. Distribution of repetitive DNA sequences in eubacteria and application to fingerprinting of bacterial genomes. Nucleic Acids Res. 1991, 19, 6823-6831. [CrossRef]

19. Silveira, L.; Nunes, A.; Pista, A.; Isidro, J.; Belo Correia, C.; Saraiva, M.; Batista, R.; Castanheira, I.; MacHado, J.; Gomes, J.P. Characterization of Multidrug-Resistant Isolates of Salmonella enterica Serovars Heidelberg and Minnesota from Fresh Poultry Meat Imported to Portugal. Microb. Drug Resist. 2021, 27, 87-98. [CrossRef] 
20. Llarena, A.; Ribeiro-Gonçalves, B.F.; Nuno Silva, D.; Halkilahti, J.; Machado, M.P.; Da Silva, M.S.; Jaakkonen, A.; Isidro, J.; Hämäläinen, C.; Joenperä, J.; et al. INNUENDO: A cross-sectoral platform for the integration of genomics in the surveillance of food-borne pathogens. EFSA Support. Publ. 2018, 15, 1498E. [CrossRef]

21. Aziz, R.K.; Bartels, D.; Best, A.; DeJongh, M.; Disz, T.; Edwards, R.A.; Formsma, K.; Gerdes, S.; Glass, E.M.; Kubal, M.; et al. The RAST Server: Rapid annotations using subsystems technology. BMC Genom. 2008, 9, 75. [CrossRef] [PubMed]

22. Rossi, M.; da Silva, M.S.; Ribeiro-Gonçalves, B.F.; Silva, D.N.; Machado, M.P.; Oleastro, M.; Borges, V.; Isidro, J.; Viera, L.; Barker, D.O.; et al. INNUENDO whole genome and core genome MLST schemas and datasets for Campylobacter jejuni. Zenodo 2018, 4 . [CrossRef]

23. Silva, M.; Machado, M.P.; Silva, D.N.; Rossi, M.; Moran-Gilad, J.; Santos, S.; Ramirez, M.; Carriço, J.A. chewBBACA: A complete suite for gene-by-gene schema creation and strain identification. Microb. Genom. 2018, 4, e000166. [CrossRef]

24. Francisco, A.P.; Bugalho, M.; Ramirez, M.; Carriço, J.A. Global optimal eBURST analysis of multilocus typing data using a graphic matroid approach. BMC Bioinform. 2009, 10, 152. [CrossRef] [PubMed]

25. Ribeiro-Gonçalves, B.; Francisco, A.P.; Vaz, C.; Ramirez, M.; Carriço, J.A. PHYLOViZ Online: Web-based tool for visualization, phylogenetic inference, analysis and sharing of minimum spanning trees. Nucleic Acids Res. 2016, 44, 246-251. [CrossRef]

26. Giacomelli, M.; Follador, N.; Coppola, L.M.; Martini, M.; Piccirillo, A. Survey of Campylobacter spp. in owned and unowned dogs and cats in Northern Italy. Vet. J. 2015, 204, 333-337. [CrossRef]

27. Amar, C.; Kittl, S.; Spreng, D.; Thomann, A.; Korczak, B.M.; Burnens, A.P.; Kuhnert, P. Genotypes and antibiotic resistance of canine Campylobacter jejuni isolates. Vet. Microbiol. 2014, 168, 124-130. [CrossRef]

28. Chaban, B.; Ngeleka, M.; Hill, J.E. Detection and quantification of 14 Campylobacter species in pet dogs reveals an increase in species richness in feces of diarrheic animals. BMC Microbiol. 2010, 10, 73. [CrossRef] [PubMed]

29. Carbonero, A.; Torralbo, A.; Borge, C.; García-Bocanegra, I.; Arenas, A.; Perea, A. Campylobacter spp., C. Jejuni and C. Upsaliensis infection-associated factors in healthy and ill dogs from clinics in Cordoba, Spain. Screening tests for antimicrobial susceptibility. Comp. Immunol. Microbiol. Infect. Dis. 2012, 35, 505-512. [CrossRef]

30. Man, S.M. The clinical importance of emerging Campylobacter species. Nat. Rev. Gastroenterol. Hepatol. 2011, 8, 669-685. [CrossRef]

31. Bourke, B.; Chan, V.L.; Sherman, P. Campylobacter upsaliensis: Waiting in the wings. Clin. Microbiol. Rev. 1998, 11, 440-449. [CrossRef] [PubMed]

32. Aspinall, S.T.; Wareing, D.R.A.; Hayward, P.G.; Hutchinson, D.N. Selective medium for thermophilic campylobacters including Campylobacter upsaliensis. J. Clin. Pathol. 1993, 46, 829-831. [CrossRef]

33. Bojanić, K.; Midwinter, A.C.; Marshall, J.C.; Biggs, P.J.; Acke, E. Isolation of emerging Campylobacter species in working farm dogs and their frozen home-killed raw meat diets. J. Vet. Diagn. Investig. 2019, 31, 23-32. [CrossRef]

34. Aspinall, S.T.; Wareing, D.R.A.; Hayward, P.G.; Hutchinson, D.N. A comparison of a new campylobacter selective medium (CAT) with membrane filtration for the isolation of thermophilic campylobacters including Campylobacter upsaliensis. J. Appl. Bacteriol. 1996, 80, 645-650. [CrossRef]

35. Santaniello, A.; Varriale, L.; Dipineto, L.; Borrelli, L.; Pace, A.; Fioretti, A.; Menna, L.F. Presence of campylobacter jejuni and C. Coli in dogs under training for animal-assisted therapies. Int. J. Environ. Res. Public Health 2021, 18, 3717. [CrossRef]

36. Lee, M.K.; Billington, S.J.; Joens, L.A. Potential virulence and antimicrobial susceptibility of Campylobacter jejuni isolates from food and companion animals. Foodborne Pathog. Dis. 2004, 1, 223-230. [CrossRef]

37. Rossi, M.; Hänninen, M.L.; Revez, J.; Hannula, M.; Zanoni, R.G. Occurrence and species level diagnostics of Campylobacter spp., enteric Helicobacter spp. and Anaerobiospirillum spp. in healthy and diarrheic dogs and cats. Vet. Microbiol. 2008, 129, 304-314. [CrossRef]

38. Sahin, O.; Burrough, E.R.; Pavlovic, N.; Frana, T.S.; Madson, D.M.; Zhang, Q. Campylobacter jejuni as a cause of canine abortions in the United States. J. Vet. Diagn. Investig. 2014, 26, 699-704. [CrossRef]

39. Revez, J.; Rossi, M.; Ellström, P.; de Haan, C.; Rautelin, H.; Hänninen, M.L. Finnish Campylobacter jejuni strains of multilocus sequence type ST-22 complex have two lineages with different characteristics. PLoS ONE 2011, 6, e26880. [CrossRef]

40. Islam, Z.; van Belkum, A.; Wagenaar, J.A.; Cody, A.J.; de Boer, A.G.; Tabor, H.; Jacobs, B.C.; Talukder, K.A.; Endtz, H.P. Comparative genotyping of Campylobacter jejuni strains from patients with Guillain-Barré syndrome. PLoS ONE 2009, 4, e7257. [CrossRef] [PubMed]

41. Taboada, E.N.; van Belkum, A.; Yuki, N.; Acedillo, R.R.; Godschalk, P.C.R.; Koga, M.; Endtz, H.P.; Gilbert, M.; Nash, J.H.E. Comparative genomic analysis of Campylobacter jejuni associated with Guillain-Barré and Miller Fisher syndromes: Neuropathogenic and enteritis-associated isolates can share high levels of genomic similarity. BMC Genom. 2007, 8, 359. [CrossRef]

42. Dingle, K.E.; Colles, F.M.; Ure, R.; Wagenaar, J.A.; Duim, B.; Bolton, F.J.; Fox, A.J.; Wareing, D.R.A.; Maiden, M.C.J. Characterization of Campylobacter jejuni Clones: A Basis for Epidemiologic Investigation. Emerg. Infect. Dis. 2002, 8, 949-955. [CrossRef] [PubMed]

43. De Haan, C.P.A.; Kivistö, R.; Hakkinen, M.; Rautelin, H.; Hänninen, M.L. Decreasing trend of overlapping multilocus sequence types between human and chicken campylobacter jejuni isolates over a decade in finland. Appl. Environ. Microbiol. 2010, 76, 5228-5236. [CrossRef] [PubMed] 